\title{
Auditory location negative priming: A case of feature mismatch
}

\author{
Susanne Mayr, Robert Hauke, and Axel Buchner \\ Heinrich-Heine-Universität Düsseldorf, Düsseldorf, Germany
}

\begin{abstract}
In an auditory four-alternative forced choice localization task, participants had to localize one of two simultaneously presented sounds while ignoring the location of the second sound. Negative priming - that is, sloweddown responses to a location that had to be ignored in the previous trial - was found only when the sound at the repeated location changed between prime and probe. There was also no increase in prime response errors to the probes of ignored repetition trials. These findings allow for the conclusion that auditory location priming is caused by feature mismatch only and that other mechanisms, such as inhibition of ignored locations or episodic retrieval of transfer-inappropriate prime information, do not play a role.
\end{abstract}

Negative priming - the slow-down in responding to previously ignored stimuli - was originally discovered in the visual domain (Neill, 1977; Tipper, 1985). Negative priming research in other modalities has been rare. However, on the basis of the limited number of studies, it can be inferred that negative priming exists in the auditory (e.g., Buchner $\&$ Steffens, 2001) and the tactile (Frings, Bader, \& Spence, 2008) modalities. At least with respect to auditory negative priming, the underlying mechanisms resemble those in the visual domain (see Mayr \& Buchner, 2007). However, all auditory negative priming studies have investigated identity negative priming tasks for which participants have to select a stimulus on the basis of a criterion such as ear (in audition) or color (in vision) and have to respond to an aspect of stimulus identity, such as categorical membership or identity. None of the auditory experiments so far have been concerned with spatial negative priming. In spatial tasks, selection is based on a criterion such as identity, but responses have to be made to the location of the stimulus (Tipper, Brehaut, \& Driver, 1990).

To explain the visuospatial negative priming effect, Park and Kanwisher (1994) suggested that the slowed-down reaction to a recently ignored object was the result of a mismatch in the representation of the repeated object between prime and probe. Their participants indicated at which of four positions a target (e.g., "o") had occurred, while ignoring a distractor (e.g., "+") at another location. In ignored repetition trials, the probe target appeared at the same location as the prime distractor. In control trials, the probe target appeared in a previously unoccupied position. Reactions in ignored repetition trials were slower than those in control trials. The authors argued that this negative priming effect was caused by a mismatch between prime and probe displays because the probe target ("o") differed from the item that occupied the same position in the prime ("+") in ignored repetition trials. When, in contrast, the prime distractor had the same identity as the probe target and both appeared at the same location, negative priming was absent. This finding is consistent with the feature mismatch hypothesis, because there was no prime-to-probe symbol change at the probe target location. It is inconsistent with distractor inhibition (Tipper, 1985) and episodic retrieval models (Mayr \& Buchner, 2006; Neill \& Valdes, 1992; Neill, Valdes, Terry, \& Gorfein, 1992). The inhibition model predicts spatial negative priming due to a suppression of the mental representation of the prime distractor location. The episodic retrieval model predicts negative priming due to retrieval of a do-not-respond tag attached to the previously ignored location or due to inappropriate retrieval of prime response information. More recent work, however, has demonstrated that feature mismatch cannot be the (sole) basis of visual location priming, because negative priming has been found in the absence of feature mismatches (e.g., Milliken, Tipper, \& Weaver, 1994). Furthermore, Buckolz, Goldfarb, and Khan (2004; cf. Guy \& Buckolz, 2007) presented evidence for a late response-related locus of spatial priming, showing that the execution of a former distractor response causes negative priming, rather than the reuse of the former distractor location as the probe target location.

For the first time, we implemented an auditory spatial priming task. Participants responded to the location of a sound while ignoring a second sound at another location. On ignored repetition trials, the subsequent target was presented at the previously ignored location. We expected to find a location negative priming effect, with slower responses to the former distractor location, relative to a control location. In analogy to the visual experiments, we tested whether feature mismatch was a major factor of auditory spatial negative priming by manipulating whether the selection criterion changed between prime and probe. For ignored repetition

S. Mayr, susanne.mayr@uni-duesseldorf.de 
trials, a selection criterion change (e.g., prime, attend to the piano; probe, attend to the (row) implies that the sound at the repeated location stays the same (match). In contrast, when there is no selection criterion change (e.g., prime/ probe, attend to the piano), the repeated location in ignored repetition trials is occupied by different sounds in the prime and probe (mismatch). If negative priming occurred only if the selection criterion did not change, this would imply that feature mismatch was the exclusive cause of auditory negative priming. If negative priming were larger in no-change (mismatch) than in change (match) trials, we would have to infer that other mechanisms, such as inhibition or episodic retrieval, are involved. Finally, if negative priming were unaffected by the selection criterion change manipulation, feature mismatch could not play a role in auditory location negative priming.

In identity priming tasks, participants make more probe errors with the former prime response in ignored repetition than in control trials (Mayr \& Buchner, 2006). This finding has been interpreted as evidence for the task-inappropriate retrieval of the prime episode cued by the repeated stimulus. In order to investigate whether this process is also involved in auditory spatial priming, we analyzed the frequency of prime response errors. If the repetition of a stimulus at a previously occupied but ignored location cues retrieval of the previous prime episode, including the prime response, there should also be an increase of prime response errors in ignored repetition trials, as compared with control trials. If the repeated presentation of an identical stimulus at the same location is necessary to retrieve the former prime episode and response, prime response errors should increase only in match trials. If, however, the repeated occupation of a location with a stimulus, irrespective of stimulus identity, is sufficient to retrieve the prime, we should find a prime response retrieval effect in match and mismatch trials.

\section{METHOD}

\section{Participants}

The data of 4 participants were eliminated due to excessive error frequencies $(>.40)$ in at least one condition. The final sample of 69 participants (56 females) ranged in age from 18 to 37 years $(M=$ 23.10). The participants were tested individually and either were paid or received course credit.

\section{Materials}

The stimuli were two digitized sounds (piano, crow). Each sound was $300 \mathrm{msec}$ long. The piano sound was composed of a base fre- quency of $440 \mathrm{~Hz}$ and three harmonics. The crow sound comprised frequencies primarily between 600 and 2,000 Hz and 2,600 and $3,600 \mathrm{~Hz}$. The participants were seated at the center of a room in front of a 17-in. iMac. Viewing distance was $60 \mathrm{~cm}$. The computer was connected to four Yamaha MSP3 loudspeakers, which were positioned as follows. Two loudspeakers were placed in front of the participant, one $40^{\circ}$ to the right of the sagittal head-computerdisplay axis, the other $40^{\circ}$ to the left. Both speakers were placed at a $1.40-\mathrm{m}$ distance from the participant's head. The remaining speakers were located in the same spatial arrangement behind the participant. Speakers were approximately at the height of the participant's ears.

Each trial consisted of a prime and a probe display. Each display consisted of a target presented to one location and a simultaneously presented distractor at one of the three other locations. A picture appearing before each display indicated the to-be-attended sound. The attended sound either changed between prime and probe (selection criterion change) or stayed the same (selection criterion no change). The participants reacted to the location of the attended sound by pressing one of four keys on the number pad of the keyboard. The key layout was spatially compatible to the locations of the loudspeakers they represented (4, front left; 5 , front right; 1 , rear left; 2 , rear right). The keys were pressed using the left and right middle and index fingers.

The ignored repetition and control trials were constructed to be parallel (Table 1). For an ignored repetition trial, prime and probe target and distractor locations were combined under two restrictions. The ignored prime location was identical to the attended probe location, and target and distractor sounds within a display were different. A parallel control trial was constructed by presenting the ignored prime sound at the only vacant location on the ignored repetition trial.

Within these two types of trials, the ignored prime location would have been the attended probe location on $50 \%$ of the trials, and attended prime and probe locations would never have been equal. We therefore added attended repetition and attended repetition control trials that allowed - as a side effect - analysis of auditory spatial repetition priming effects and their dependence on feature mismatch. Attended repetition trials were constructed by selecting three of the four locations as prime and probe target and distractor locations, with the restriction that the attended prime location had to be identical to the attended probe location. Attended repetition control trials were constructed by presenting the attended prime sound at the only vacant location on the parallel attended repetition trial.

Note that an ignored repetition trial always shared its control trial with an attended repetition trial (see Table 1 for an example). Had we used the entire set of trials that can be generated by the algorithm just described, every control trial would have occurred twice, possibly resulting in an unwanted response speedup at the second presentation of each control trial (which would artificially increase the negative priming effect in reaction times). In order to avoid this confound for control trials, ignored and attended repetition trials were systematically assigned to Set 1 or 2, with three restrictions. First, identical control and attended repetition control trials had to belong to different sets. Second, within each trial type, the frequencies of occupied locations had to be identical. Third, the frequencies of the combinations of attended and ignored locations, both within the prime and within

Table 1

Examples of Stimulus Configurations for Trials

With Selection Criterion Change Between Prime and Probe

\begin{tabular}{|c|c|c|c|c|c|c|c|c|c|}
\hline & \multirow[b]{2}{*}{ Location } & \multicolumn{2}{|c|}{$\begin{array}{l}\text { Ignored } \\
\text { Repetition }\end{array}$} & \multicolumn{2}{|c|}{ Control } & \multicolumn{2}{|c|}{$\begin{array}{l}\text { Attended } \\
\text { Repetition }\end{array}$} & \multicolumn{2}{|c|}{$\begin{array}{c}\text { Attended } \\
\text { Repetition Control }\end{array}$} \\
\hline & & Left & Right & Left & Right & Left & Right & Left & Right \\
\hline Prime & $\begin{array}{l}\text { Front } \\
\text { Rear }\end{array}$ & piano & crow & piano & crow & $\begin{array}{l}\text { crow } \\
\text { piano }\end{array}$ & & piano & crow \\
\hline Probe & $\begin{array}{l}\text { Front } \\
\text { Rear }\end{array}$ & piano & crow & piano & crow & piano & crow & piano & crow \\
\hline
\end{tabular}

Note-Attended locations are indicated by boldface type. Left, right, front, and rear represent the locations of the four loudspeakers relative to the participant. 
the probe pairs, had to be equal for the different trial types. For each set, the attended probe location could not be inferred from the prime display. The participants were randomly assigned to the sets.

Each set included 12 trials of each of the four types (ignored repetition, control, attended repetition, attended repetition control). Each of the trials was presented once with and once without a prime-toprobe selection criterion change. Furthermore, each of these trials was presented once starting with the piano as the attended prime stimulus sound and once with the crow. Consequently, each set comprised 192 unique trials $(4 \times 12 \times 2 \times 2)$, which were presented twice. The 384 experimental trials were presented in a random sequence.

\section{Procedure}

The participants first adjusted the loudness to a comfortable level that nevertheless enabled them to differentiate the sounds (mode, $64 \mathrm{db}[\mathrm{A}]$; range, 54-69 db[A]). Training trials followed. The experiment began only after the participants had responded correctly on $70 \%$ of the past 15 trials of, at most, 50 trials.

Each experimental trial began with the visual presentation of the selection criterion (piano or crow) that had to be localized subsequently. After a 500-msec cue-target interval, prime sounds were presented. With the prime reaction, the selection criterion display was removed. A 1,000-msec prime-probe interval preceded onset of the probe selection criterion display. Probe sounds were presented $500 \mathrm{msec}$ later. Reactions faster than $100 \mathrm{msec}$ and slower than 3,000 msec were counted as invalid. The participants received audiovisual feedback about the correctness of their reactions. A 1,000 -msec intertrial interval preceded the next trial.

After every 12 th trial, the participants received a summary feedback about their error percentage and average reaction time. After the final trial, all the participants were informed about the purpose of the experiment.

\section{Design}

The experiment comprised a $2 \times 2$ design with trial type (ignored repetition vs. control) and selection criterion (change vs. no change) as within-subjects variables and another $2 \times 2$ design with trial type (attended repetition vs. control) and selection criterion (change vs. no change) as within-subjects variables. The primary dependent variable was participants' average reaction times, but error rates were also analyzed.

A difference of $f=0.25$ in the size of the negative priming effect (ignored repetition - control) between the selection criterion change and the no-change condition could be detected with a power of $1-\beta=.93$, given a population correlation of $\rho=.3$ between the reaction time differences in the two conditions, $N=69$ participants, and $\alpha=.05$ (Faul, Erdfelder, Lang, \& Buchner, 2007).

\section{RESULTS}

Probe reaction times were evaluated for trials with correct prime and probe responses. For the ignored repetition design (Figure 1, left side), the trial type manipulation was modulated by selection criterion: Responses to ignored repetition trials were facilitated in the change condition but slowed down in the no-change condition.

In the $2 \times 2$ MANOVA of the reaction time data, with trial type (ignored repetition vs. control) and selection criterion (change vs. no change) as within-subjects variables, the main effects of trial type and selection criterion were not statistically significant $\left[F(1,68)=3.31, p=.07, \eta^{2}=.05\right.$, and $F(1,68)=2.10, p=.15, \eta^{2}=.03$, respectively], but the interaction was significant $[F(1,68)=19.21, p<.01$, $\left.\eta^{2}=.22\right]$. For change trials, responses in ignored repetition trials were faster than those in control trials, but a positive priming effect fell short of significance $[t(68)=-1.95$,

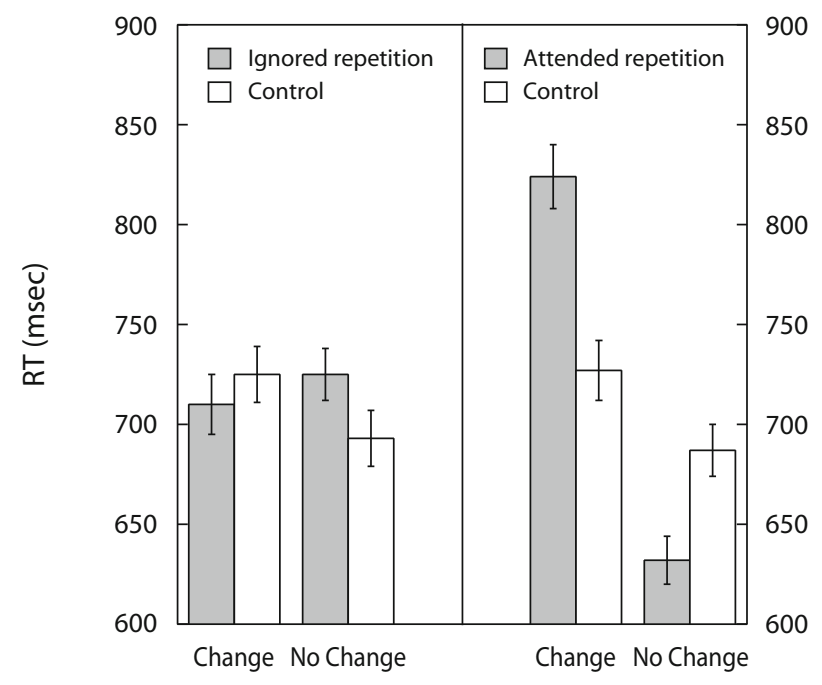

Selection Criterion

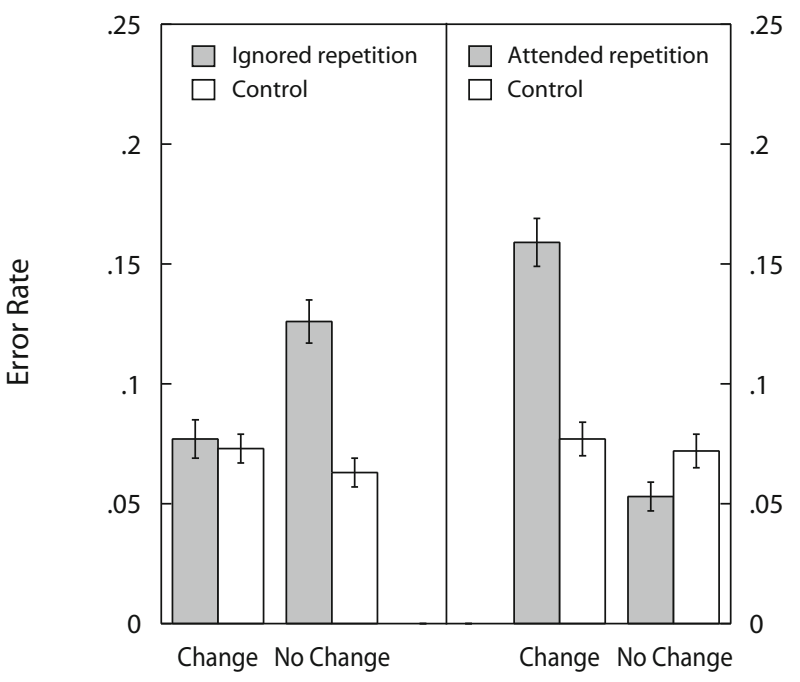

Selection Criterion

Figure 1. Sample average of participants' mean reaction times (RTs; upper panel) and error rates (lower panel) as a function of trial type and selection criterion. The error bars depict the standard errors of the means.

$p=.06$ (all follow-up $t$ tests are two-tailed), $\left.d_{\mathrm{z}}=0.23\right]$. For no-change trials, there was a significant negative priming effect $\left[t(68)=4.93, p<.01, d_{\mathrm{z}}=0.59\right]$.

An analysis of the error data showed significant main effects of trial type $\left[F(1,68)=41.93, p<.01, \eta^{2}=.38\right]$ and selection criterion $\left[F(1,68)=15.94, p<.01, \eta^{2}=.19\right]$ and a significant interaction $\left[F(1,68)=27.16, p<.01, \eta^{2}=\right.$ .29]. Negative priming was not significant for change trials $\left[t(68)=0.58, p=.56, d_{\mathrm{z}}=0.07\right]$ but was significant for no-change trials $\left[t(68)=7.39, p<.01, d_{\mathrm{z}}=0.89\right]$.

For the attended repetition design (Figure 1, right side), a $2 \times 2$ MANOVA of the reaction time data with trial type (attended repetition vs. control) and selection criterion (change vs. no change) as within-subjects variables showed signifi- 
Table 2

\begin{tabular}{|c|c|c|c|c|}
\hline \multicolumn{5}{|c|}{$\begin{array}{c}\text { Accumulated Absolute Frequencies of Correct Probe Responses and of the Different } \\
\text { Types of Probe Errors As a Function of Trial Type (Ignored Repetition vs. Control) } \\
\text { and Selection Criterion (Change vs. No Change) }\end{array}$} \\
\hline & \multicolumn{2}{|c|}{$\begin{array}{c}\text { Selection Criterion } \\
\text { Change Trials }\end{array}$} & \multicolumn{2}{|c|}{$\begin{array}{l}\text { Selection Criterion } \\
\text { No Change Trials }\end{array}$} \\
\hline & $\begin{array}{c}\text { Ignored } \\
\text { Repetition }\end{array}$ & Control & $\begin{array}{l}\text { Ignored } \\
\text { Repetition }\end{array}$ & Control \\
\hline orrect probe target location responses & 2,837 & 2,844 & 2,711 & 2,899 \\
\hline ncorrect probe distractor location responses & 136 & 138 & 212 & 104 \\
\hline Incorrect prime target location responses & 39 & 30 & 87 & 45 \\
\hline Other incorrect responses* & 54 & 51 & 84 & 40 \\
\hline
\end{tabular}

*Ignored repetition trials, incorrect responses using the key that was assigned to the vacant stimulus position; control trials, incorrect prime distractor position responses.

cant main effects of trial type $[F(1,68)=12.66, p<.01$, $\left.\eta^{2}=.16\right]$ and selection criterion $[F(1,68)=334.97, p<.01$, $\left.\eta^{2}=.83\right]$. The interaction was also significant $[F(1,68)=$ $\left.264.05, p<.01, \eta^{2}=.80\right]$. For change trials, reactions to attended repetition trials were significantly slower than those to control trials $\left[t(68)=11.84, p<.01, d_{\mathrm{z}}=1.43\right]$. For nochange trials, there was a significant positive priming effect $\left[t(68)=8.16, p<.01, d_{\mathrm{z}}=0.98\right]$.

An analysis of the error data showed significant main effects of trial type $\left[F(1,68)=38.83, p<.01, \eta^{2}=.36\right]$ and selection criterion $\left[F(1,68)=99.86, p<.01, \eta^{2}=\right.$ $.60]$ and a significant interaction $[F(1,68)=75.97, p<$ $\left..01, \eta^{2}=.53\right]$. For change trials, more errors were committed in attended repetition than in control trials $[t(68)=$ $\left.9.84, p<.01, d_{\mathrm{z}}=1.18\right]$. In contrast, there was a significant positive priming effect for no-change trials $[t(68)=$ 2.71, $p=.01, d_{\mathrm{z}}=0.33$ ].

For the error frequency data displayed in Table 2, we estimated the probability of prime response errors in probe trials, using the multinomial model introduced by Mayr and Buchner (2006). Parameters $p r r_{\mathrm{IR}}$ and $p r r_{\mathrm{C}}$ represent the conditional probabilities of committing a prime response error (given neither a correct response nor a probe discrimination error) for the ignored repetition and control trials, respectively. Finding that ${ } r r_{\mathrm{IR}}$ was larger than $\mathrm{prr}_{\mathrm{C}}$ would indicate that the probe presentation cues retrieval of the inappropriate prime response. Figure 2 shows that the probability estimates for both parameters did not differ between ignored repetition and control trials, irrespective of selection criterion condition. This was confirmed by the fact that the restricted model with $p r r_{\mathrm{IR}}=p r r_{\mathrm{C}}$ fitted the data for the change condition $\left[G^{2}(1)=0.43, p=.51\right]$, as well as for the no-change condition $\left[G^{2}(1)=0.10, p=.76\right]$.

\section{DISCUSSION}

The results of this experiment can be summarized as follows. First, a spatial negative priming effect was found. To our knowledge, this is the first report of spatial negative priming in the auditory domain. What is more, the effect was present only on trials for which the sound at the ignored prime location changed when that location had to be attended in the probe. In other words, negative priming depended on whether feature mismatch occurred at the repeated location. In contrast, when the same stimulus oc- cupied the repeated location in selection criterion change trials, there was no feature mismatch and, thus, no negative priming effect. Quite to the contrary, responses to ignored repetition trials in the change condition were accompanied by a small (but not significant) positive priming effect.

The disordinal interaction of the trial type and selection criterion manipulation disproves an explanation in terms of inhibition (Tipper, 1985) or episodic retrieval (Neill \& Valdes, 1992; Neill et al., 1992). Otherwise, we should have found a negative priming effect for both selection criterion conditions. Instead, the observed exclusive dependence of negative priming on feature mismatch at the repeated location is compatible with early findings in the visual domain (Park \& Kanwisher, 1994). However, the preponderance of studies argues against a feature mismatch account of visuospatial priming and for an additional influence of either inhibition or episodic retrieval processes (e.g., Milliken, Tip-



Figure 2. Probability estimates for the model parameters representing the probability of prime response retrieval as a function of trial type (ignored repetition vs. control) and selection criterion. The error bars depict the standard errors of the parameter estimates. 
per, Houghton, \& Lupiáñez, 2000; Milliken et al., 1994) and a response locus of spatial negative priming (Buckolz et al., 2004). On the basis of the finding reported here, the involvement of processes other than feature mismatch in auditory spatial priming waits for substantiation.

With respect to our attended repetition trials, responding was facilitated when identical sounds were presented at the same location but was impaired when different sounds had to be localized. In other words, responding was entirely determined by physical (mis)matches at the repeated location, but not by inhibition of return (Posner $\&$ Cohen, 1984) to a previously attended location.

The modulating effects of the match/mismatch manipulation were stronger for the attended repetition than for the ignored repetition trials. Possibly, repeated attention to a location increases the effect of a (mis)match detection. However, it is also conceivable that detection of a mismatch at a repeatedly attended location interfered not only at the level of perceptual processing, but also at a later processing level. In accordance with the bypass rule (Fletcher \& Rabbitt, 1978; Krueger \& Shapiro, 1981), participants are inclined to repeat a response if the stimuli are the same across adjacent trials (due to the formation of a direct link from early stimulus representation to the response; see Pashler $\&$ Baylis, 1991), but they will tend to change the response if the stimuli differ. The bypass rule can be applied to our attended repetition findings: The participants were slowed down (as compared with control) when they repeated their response but the stimulus changed, and they responded faster when the stimulus and response stayed the same. Consequently, the origin of our attended repetition effects (perceptual and/or postperceptual) is ambiguous.

An increase in prime response errors has been interpreted as evidence for a prime-response retrieval mechanism in identity negative priming (Mayr \& Buchner, 2006). There was no evidence for such a mechanism in the change or the no-change condition. The absence of an increase in prime-response retrieval fits well with the reaction time and overall error data. Two possibilities come to mind as to why the repeated occupation of a location-first as distractor, then as target location - may not result in retrieval of the former prime episode. First, the retrieval cue of the spatial location occupied by a sound may not be effective, not even when this sound is identical (as in match trials). Second, action-oriented episodes may not be stored in the case of location priming. In fact, the auditory spatial negative priming effect in mismatch trials shows that memory of the prime episode must be involved, at least as far as the physical attributes of the stimulus at the specific location are concerned. However, this memory does not seem to include attributes of the prime response.

In sum, the present findings establish location-based negative priming in the auditory modality as an empirical phenomenon, and they suggest that auditory spatial negative priming is entirely determined by physical feature mismatches.

\section{AUTHOR NOTE}

The research reported in this article was supported by a grant from the Deutsche Forschungsgemeinschaft (Ma 2610/2-1). Correspondence concerning this article should be addressed to S. Mayr, Institut für Experimentelle Psychologie, Heinrich-Heine-Universität, 40225 Düsseldorf, Germany (e-mail: susanne.mayr@uni-duesseldorf.de).

\section{REFERENCES}

Buchner, A., \& Steffens, M. C. (2001). Auditory negative priming in speeded reactions and temporal order judgments. Quarterly Journal of Experimental Psychology, 54A, 1125-1142.

Buckolz, E., Goldfarb, A., \& Khan, M. (2004). The use of a distractorassigned response slows later responding in a location negative priming task. Perception \& Psychophysics, 66, 837-845.

Faul, F., Erdfelder, E., Lang, A.-G., \& Buchner, A. (2007). $\mathrm{G}^{*}$ Power 3: A flexible statistical power analysis program for the social, behavioral, and biomedical sciences. Behavior Research Methods, 39, 175-191.

Fletcher, B. C., \& RabittT, P. M. (1978). The changing pattern of perceptual analytic strategies and response selection with practice in a two-choice reaction time task. Quarterly Journal of Experimental Psychology, 30, 417-427.

Frings, C., Bader, R., \& Spence, C. (2008). Selection in touch: Negative priming with tactile stimuli. Perception \& Psychophysics, 70, 516-523.

GuY, S., \& Buckolz, E. (2007). The locus and modulation of the location negative priming effect. Psychological Research, 71, 178-191.

Krueger, L. E., \& Shapiro, R. G. (1981). Intertrial effects of samedifferent judgments. Quarterly Journal of Experimental Psychology, 33A, 241-265.

MAYR, S., \& Buchner, A. (2006). Evidence for episodic retrieval of inadequate prime responses in auditory negative priming. Journal of Experimental Psychology: Human Perception \& Performance, 32, 932-943.

MaYr, S., \& Buchner, A. (2007). Negative priming as a memory phenomenon: A review of 20 years of negative priming research. Zeitschrift für Psychologie, 215, 35-51.

Milliken, B., Tipper, S. P., Houghton, G., \& Lupiáñez, J. (2000). Attending, ignoring, and repetition: On the relation between negative priming and inhibition of return. Perception \& Psychophysics, 62, 1280-1296.

Milliken, B., TipPer, S. P., \& Weaver, B. (1994). Negative priming in a spatial localization task: Feature mismatching and distractor inhibition. Journal of Experimental Psychology: Human Perception \& Performance, 20, 624-646.

NeILL, W. T. (1977). Inhibitory and facilitatory processes in selective attention. Journal of Experimental Psychology: Human Perception \& Performance, 3, 444-450.

Neill, W. T., \& VAldes, L. A. (1992). Persistence of negative priming: Steady state or decay? Journal of Experimental Psychology: Learning, Memory, \& Cognition, 18, 565-576.

Neill, W. T., Valdes, L. A., Terry, K. M., \& Gorfein, D. S. (1992). Persistence of negative priming: II. Evidence for episodic trace retrieval. Journal of Experimental Psychology: Learning, Memory, \& Cognition, 18, 993-1000.

PARK, J., \& KANWISHER, N. (1994). Negative priming for spatial locations: Identity mismatching, not distractor inhibition. Journal of Experimental Psychology: Human Perception \& Performance, 20, 613-623.

Pashler, H. E., \& BaYlis, G. C. (1991). Procedural learning: II. Intertrial repetition effects in speeded-choice tasks. Journal of Experimental Psychology: Learning, Memory, \& Cognition, 17, 33-48.

Posner, M. I., \& Cohen, Y. (1984). Components of attention. In H. Bouma \& D. Bowhuis (Eds.), Attention and performance $X$ (pp. 531-556). Hillsdale, NJ: Erlbaum.

TIPPER, S. P. (1985). The negative priming effect: Inhibitory priming by ignored objects. Quarterly Journal of Experimental Psychology: Human Experimental Psychology, 37A, 571-590.

Tipper, S. P., Brehaut, J. C., \& Driver, J. (1990). Selection of moving and static objects for the control of spatially directed action. Journal of Experimental Psychology: Human Perception \& Performance, 16, 492-504.

(Manuscript received September 15, 2008; revision accepted for publication April 22, 2009.) 